\title{
Influence of liquid properties on ultrasonic atomization
}

\author{
A. Lozano*, J.A. García, J. Alconchel, F. Barreras, E. Calvo, J.L. Santolaya \\ LIFTEC, CSIC - Universidad de Zaragoza, Zaragoza, Spain \\ ${ }^{*}$ Corresponding author: alozano@litec.csic.es
}

\begin{abstract}
Ultrasonic atomization is very convenient because it can generate droplets with diameters of a few microns and with very narrow size distribution. Besides, opposite to twin fluid nozzles, in ultrasonic atomization, droplet generation and transport are decoupled processes. Droplets are ejected from the liquid surface with very low velocities, so driving them is relatively simple. Although this atomization method is now common in some specific applications, for example in household humidifiers, there are still some details about the physics of this process that are not completely understood. Up to date, most of the published results have been limited to experiments with water. However, it has been demonstrated that atomization rates quickly decrease as liquid viscosity increases. This work analyzes the characteristics of ultrasonic atomization of some alternative fluids to determine if there is any influence of other physical properties such as surface tension or vapor pressure. Experiments are performed using a commercial piezoceramic disk with a resonance frequency of $1.65 \mathrm{MHz}$. The disk is excited with a sinusoidal signal with voltage amplitudes that go up to $60 \mathrm{~V}$. Sprays are visually characterized analyzing instantaneous images and high speed video sequences. Besides atomization rates are calculated by measuring the weight loss in a fixed time.
\end{abstract}

\section{Keywords}

Ultrasonic atomization, ultrasound, piezocramic.

\section{Introduction}

Ultrasonic atomization has some unique characteristics that are ideal for many specific applications. Typical examples are most commercial household air humidifiers, or some inhalers for drug delivery to the lungs. In these devices, atomization is achieved by vibration of an ultrasonic transducer submerged in a liquid volume. As a result, droplets can be generated from the liquid surface with diameters of a few microns, with very narrow size distribution, and with low velocity. Opposite to pressure and twin fluid nozzles, in which small droplet diameters are associated to high liquid and gas velocities, in ultrasonic atomization, droplet generation and transport are decoupled processes. Droplets are ejected from the liquid surface with very low velocity, so driving them is relatively simple.

The possibility to generate a cloud of droplets by means of ultrasonic waves was first reported by Wood and Loomis in 1927 [1]. Since then, many theoretical and experimental works have been published to explain the physics controlling this phenomenon. Two main mechanisms are considered to be responsible for the spray formation: cavitation inside the liquid mass and instabilities of standing waves on its free surface. Droplet detachment from wave crests can clearly be observed for low excitation frequencies but cannot be distinguished for frequencies in the $\mathrm{MHz}$ range. The importance of cavitation might be dependent on the forcing frequency or the ultrasonic power, but these extremes have not been demonstrated in a definite way. As confirmed by numerous experiments, it is now generally accepted that, in ultrasonic atomization, spray mean droplet diameter is essentially determined only by the oscillation frequency. The oscillation amplitude controls the spray flow rate but does not have a major influence on drop diameter [2]. When using ultrasonic transducers this amplitude is proportional to the driving signal voltage.

A major part of the published results are limited to water atomization. The influence of fluid physical properties has been studied in a relative low number of papers [3]. Most of them are based on experimental considerations [4,5], although some theoretical analysis can also be found [6]. Furthermore, in some of these works, the ultrasonic frequency is low [7]. Liquid viscosity does not substantially alter the droplet size distribution, but has a dramatic effect on atomization rate [8]. With the specific conditions in the present experiments, efficiency drops dramatically for kinematic viscosity values over $3 \times 10-6 \mathrm{~m}^{2} / \mathrm{s}$. This is a severe limitation that can preclude the use of this atomization method in many processes of industrial interest, for example in surface coating, or to introduce the droplets in a chemical reactor. However mists of viscous liquids can be obtained diluting them first in volatile solvents and atomizing the low viscosity mixture [9]. Once the solvent has evaporated, the result is a mist of a liquid that otherwise could not have been directly nebulized. To facilitate this possibility and advance in the 
understanding of the dependence on different physical properties, this work analyzes the characteristics of ultrasonic atomization of some organic compounds. In particular, several alkanes and allcohols are considered. Results are compared to those obtained atomizing water in the same conditions. Measured parameters include atomization rate and droplet size distribution. Visual aspect of the sprays is also examined, recording instant images and high speed sequences.

\section{Material and methods}

In the experiments here described, atomization was performed using commercially available inexpensive ultrasonic transducers as the one shown in Fig. 2. They consist of lead zirconate titanate PZT-4 piezoceramic disks with a diameter of $20 \mathrm{~mm}$, a thickness of $1.3 \mathrm{~mm}$ and a measured resonance frequency of $1.65 \mathrm{MHz}$.

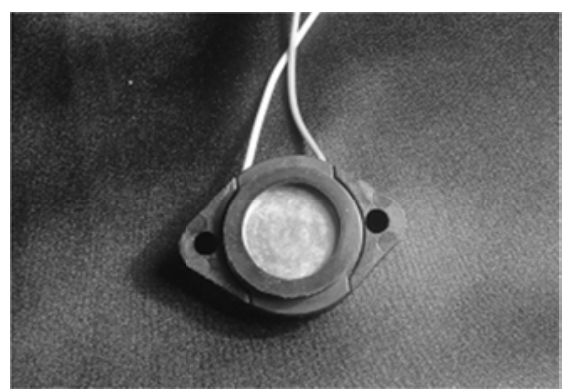

Figure 1. Image of one of the piezoceramic disks used in the present experiments

The disks were excited with a sinusoidal wave coincident with the resonance frequency and variable amplitude. It has to be noted that disks of this type only oscillate with significant amplitude when the excitation wave coincides with the main resonance peak which is quite narrow. The resonant frequency depends on the ceramic type and the disk geometry, and this is the only frequency for which atomization occurs efficiently. A scheme of the oscillator circuit used to force the disks is presented in Fig. 2. It is very important to be sure that the disks operate submerged into the fluid volume, because in this way, the liquid also acts as a cooling medium preventing the disk from overheating. Otherwise, the Curie temperature, defined as the point where the material undergoes a transition from ferromagnetic to paramagnetic, might be reached. In this case, the magnetic moments would become randomly oriented, and the ceramic element would depolarize losing its electrostrictive properties. As the disk heating increases with the applied voltage, the maximum delivered value was limited to $80 \mathrm{~V}$ to avoid damaging the ceramic. Values over $100 \mathrm{~V}$ would also risk the integrity of the power transistor in the electronic oscillator circuit (marked as T in Fig. 2).

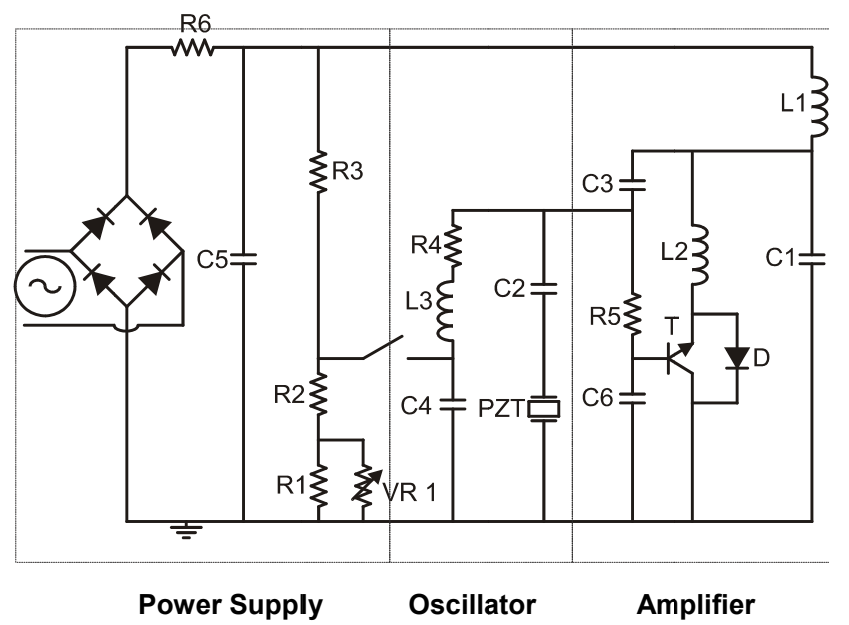

Figure 2. Scheme of the oscillator circuit

The disks were attached to the bottom plate of two different open vessels. The first one has a section of $18 \mathrm{~cm} \mathrm{x}$ $18 \mathrm{~cm}$ and a height of $24 \mathrm{~cm}$. It was used for the atomization rate measurements. A relatively large section was chosen to avoid a noticeable liquid heating from the piezoceramic transducer. The second one is even larger, with dimensions of $36 \mathrm{~cm} \times 36 \mathrm{~cm} \times 24 \mathrm{~cm}$. This one was used for the image recording, to minimize possible interferences from the walls. 
To determine the atomization rates, the decrease in the liquid mass inside the vessel during a minimum time interval of 5 minutes was monitored by weighting it with a Kern FCB scales, capable of measuring a maximum mass of $8 \mathrm{~kg}$ with a precision of $0.1 \mathrm{~g}$. For all the measurements the initial liquid level was identical and equal to 3 $\mathrm{cm}$. Care was taken to evacuate the liquid droplets out of the atomizer to prevent condensation, suctioning them with an extraction fan. This extraction, however, was limited to the small droplets that form the spray mist and not to other larger drops. This group includes, for example, splashing caused by falling of the liquid cone tip onto the pool surface. This is mainly because these drops are not useful for most applications requiring small droplets where ultrasonic atomization could be a first choice. Besides, they can be filtered out in a relatively simple manner. This study will, thus, focus on the micron-sized droplets resulting from the ultrasonic atomization.

Droplet size distributions were measured with a Malvern Mastersizer S laser difractometer equipped with a 300 $\mathrm{mm}$ focal length lens. According to the manufacturer specifications, this lens is suitable to cover a droplet diameter range from $0.5 \mu \mathrm{m}$ to $900 \mu \mathrm{m}$. The maximum obscuration in the Malvern measurements was lower than $25 \%$, with a minimum of $3.6 \%$. The room was darkened to maximize the contrast for low obscuration values. To calculate the droplet size distribution, the polydisperse model of the Malvern software was selected. The small droplets were driven to the laser beam dragging them with an air flow. To visualize the atomization process, instantaneous images were acquired with a Hamamatsu 1,024 x 1,344 pixels 12-bit C4742-95-12 ORCA-ER CCD camera with a Sigma $70-200 \mathrm{~mm}$ zoom lens. Exposure time was set to $10 \mathrm{~ms}$, and the covered field of view was $90 \mathrm{~mm} \times 118 \mathrm{~mm}(87.8 \mu \mathrm{m} / \mathrm{pixel})$. Image sequences were also acquired with a high speed CMOS RedLake Motion Pro HS4 camera, capable of recording 5,000 frames per second (fps) at a maximum image size of $512 \mathrm{x}$ 512 pixels. Two different types of sequences were registered. The first one corresponds to a field of view of 130 $\mathrm{mm} \times 130 \mathrm{~mm}$, recording speed of $5000 \mathrm{fps}$ and exposure time varying between $50 \mu \mathrm{s}$ and $150 \mu \mathrm{s}$. The second configuration corresponds to close ups with a field of view reduced to $4.2 \mathrm{~mm} \times 4.2 \mathrm{~mm}$, recording speed of 3000 fps and exposure time of $330 \mu \mathrm{s}$. To achieve this magnification $(8.2 \mu \mathrm{m} / \mathrm{pixel})$, a Nikon PB6 bellows focusing attachment was placed between the camera and the lens, together with a set of three Kenko extension rings. Two $500 \mathrm{~W}$ halogen lamps were used as illumination source.

Experiments were performed atomizing several organic compounds as well as pure water. Table 1 summarizes the values of several physical properties that can be influential in the atomization process. Liquid selection includes three alkanes and three alcohols. Density and surface tension values are quite similar for all of them, in all cases lower than those for water. Viscosity and vapor pressure cover a wider range, increasing for increasing number of $\mathrm{C}$ atoms.

Table1. Physical properties of the atomized liquids

\begin{tabular}{lcrlrrr}
\hline & $\begin{array}{l}\text { Density } \rho \\
\left(\mathrm{kg} / \mathrm{m}^{3}\right) 20^{\circ} \mathrm{C}\end{array}$ & $\begin{array}{l}\text { Viscosity } v \\
\left(\mathrm{~m}^{2} / \mathrm{s}\right) 20^{\circ} \mathrm{C}\end{array}$ & $\begin{array}{l}\text { Surface } \\
\text { tension } \sigma \\
(\mathrm{N} / \mathrm{m}) 20^{\circ} \mathrm{C}\end{array}$ & $\begin{array}{l}\text { Vapor } \\
\text { pressure } \\
(\mathrm{Pa}) 25^{\circ} \mathrm{C}\end{array}$ & $\begin{array}{l}\text { Compressibility } \\
\text { modulus K }(\mathrm{Pa}) \\
20^{\circ} \mathrm{C}\end{array}$ & $\begin{array}{l}\text { Boiling } \\
\text { Temperature } \\
\left({ }^{\circ} \mathrm{C}\right)\end{array}$ \\
\hline Water & 998.2 & $1 \times 10^{-6}$ & 0.0728 & $3.167 \times 10^{3}$ & $22.0 \times 10^{8}$ & 100 \\
Hexane & 654.8 & $0.294 \times 10^{-6}$ & 0.01843 & $20.4 \times 10^{3}$ & $7.9 \times 10^{8}$ & 69 \\
Heptane & 683.8 & $0.408 \times 10^{-6}$ & 0.0197 & $6.06 \times 10^{3}$ & $9.4 \times 10^{8}$ & 98 \\
Decane & 730.0 & $0.92 \times 10^{-6}$ & 0.02337 & $0.185 \times 10^{3}$ & $11.0 \times 10^{8}$ & 174 \\
Methanol & 781.8 & $0.745 \times 10^{-6}$ & 0.02261 & $16.96 \times 10^{3}$ & $8.23 \times 10^{8}$ & 65 \\
Ethanol & 789.0 & $1.36 \times 10^{-6}$ & 0.0228 & $7.924 \times 10^{3}$ & $8.94 \times 10^{8}$ & 78 \\
2-Propanol & 786.3 & $3.05 \times 10^{-6}$ & 0.022 & $6.02 \times 10^{3}$ & $7.5 \times 10^{8}$ & 82
\end{tabular}

\section{Results and discussion}

Before presenting and discussing the results obtained from the different measurements, it can be interesting to describe the ultrasonic atomization process. It takes place according to the following scheme. The piezoceramic disk submerged below the fluid surface starts vibrating when excited with a $1.65 \mathrm{MHz}$ sinusoidal wave. For low voltages the only noticeable effect is the appearance of some waves on the liquid surface over the disk. As the voltage is increased, this part of the surface assumes a conical shape most likely induced by an acoustic streaming phenomenon [10]. A further increase causes the elongation of the cone that forms a stem with a neck zone. Eventually, the tip of the cone detaches, and falls on the liquid pool forming big droplets due to splashing. When voltage surpasses a determinate value that depends on the liquid to be atomized and its viscosity, superimposed both to the whole mass displacement that produces the conical shape and the interfacial waves, a fine mist of small micron-sized droplets is generated, particularly in the middle part of the cone region. Together with it, some medium sized droplets are also ejected from the cone surface. The amount of this last type of droplets and their detachment velocity clearly increase with increasing forcing voltage. All these steps are 
illustrated in the series of images in Fig. 3, where water has been used as the atomized fluid. Voltages are $20 \mathrm{~V}$, $30 \mathrm{~V}, 45 \mathrm{~V}$ and $55 \mathrm{~V}$.

It has to be remarked that although the response of the CCD camera is linear, to better discern low intensity details, the images are not displayed with a linear look-up table, but with a gamma $\gamma=2$ grey scale according to the expression

$I_{\text {corr }}=I_{\max }\left(\frac{I}{I_{\max }}\right)^{1 / \gamma}$

where $I_{\text {corr }}$ are the intensity corrected values, and $I_{\max }$ is the maximum intensity.
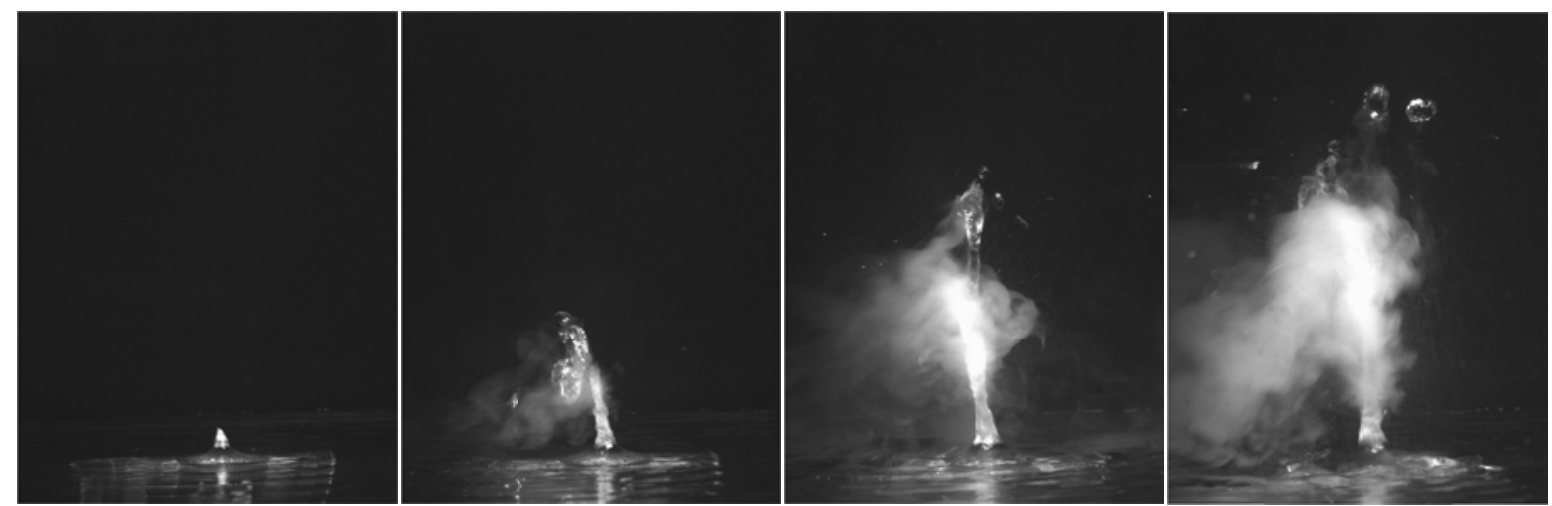

Figure 3. Evolution of the ultrasonic atomization process for increasing voltages. From left to right: $20 \mathrm{~V}, 30 \mathrm{~V}, 45 \mathrm{~V}$ and $55 \mathrm{~V}$. Atomized liquid is water.

\section{Atomization rate measurements}

A compilation of the results of the atomization rate measurements is presented in Fig. 4. From it, a first conclusion is noticeable. Water exhibits a trend that differs from the rest of liquids. While water atomization rate keeps increasing with voltage, all the other curves present a maximum for an intermediate voltage value, which varies between $35 \mathrm{~V}$ and $40 \mathrm{~V}$. Although maximum voltage in this plot is limited to $60 \mathrm{~V}$, values up to $80 \mathrm{~V}$ where tested without finding a maximum in the water graph. It is possible that still higher voltages could be required to reach it, but they would be out of the limits set for these experiments, as explained in Section 2. Setting aside water as a special case, the relative atomization rate values for the alkanes and alcohols have an inverse correlation with the kinematic viscosity, as shown in Fig. 5. Here, the maximum atomization rate for each liquid has been plotted as a function of the respective viscosity value. Again, this is in good agreement with the results in [8].

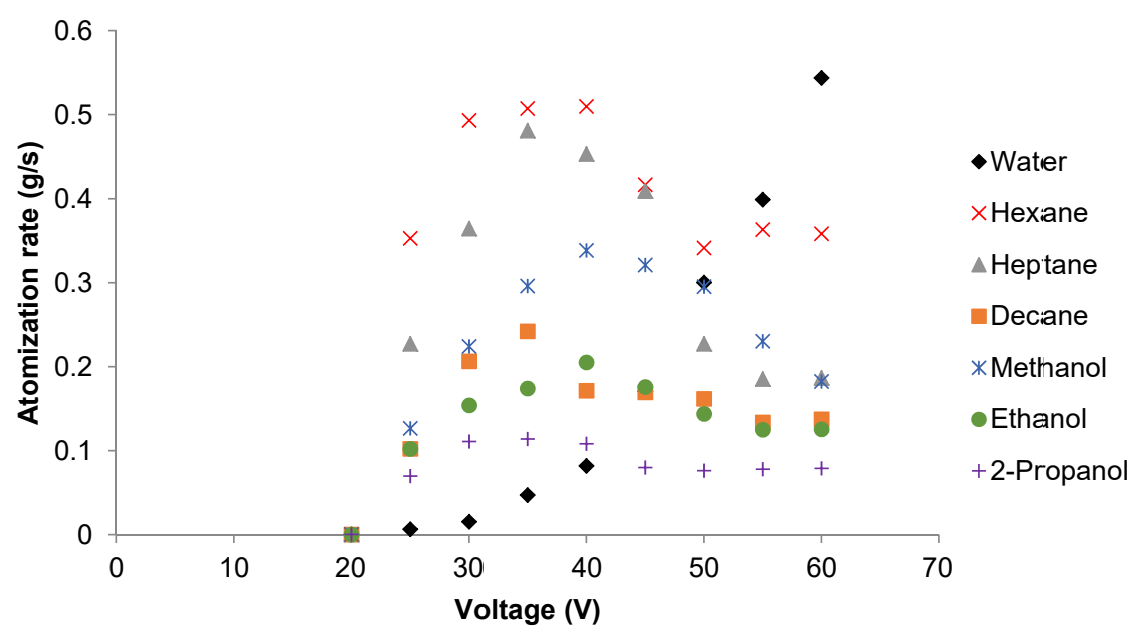

Figure 4. Atomization rate as a function of the forcing voltage for the different working liquids. 


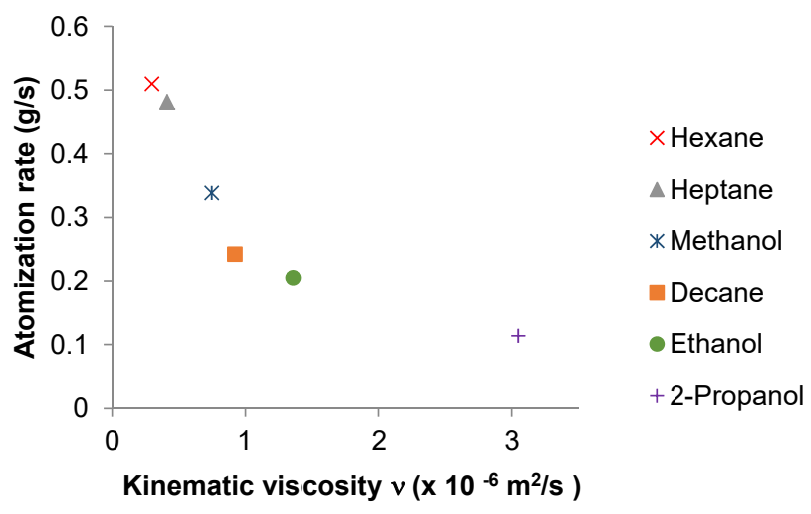

Figure 5. Maximum atomization rate for each one of the liquids as a function of the corresponding kinematic viscosity.

\section{Image analysis}

In order to confirm the results in Fig. 4, an analysis of instantaneous images of the atomization process has been performed. Figure 6 shows the situation for the different liquids at a common voltage of $30 \mathrm{~V}$. As in Fig. 3, images are displayed with a $\gamma=2$ look-up table. While for hexane, heptane and methanol the mist of small droplets is clearly visible, for decane, ethanol and water, this production is still in its initial stages. In the case of 2-propanol these droplets are almost not generated at all. Visual estimation of atomization intensity is in perfect agreement with the atomization rate values plotted in Fig. 4. From analysis of images obtained at different voltages, it can be concluded that production of the micron-sized droplets seems to be independent of the formation of the coneshaped liquid fountain. Fountain height increases with voltage, but mist formation is inhibited for high viscosity values. It is interesting to note that according to the experiments in [8] working with mixtures of glycerol and water, ultrasonic atomization is difficult for kinematic viscosities over $3 \times 10-6 \mathrm{~m}^{2} / \mathrm{s}$. Viscosity of 2-propanol, for which fog production is very low, is slightly above this limit.

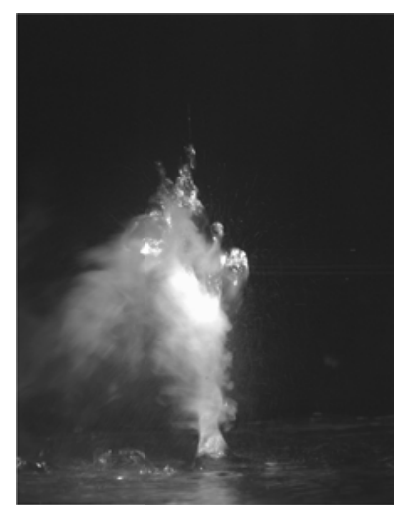

Hexane

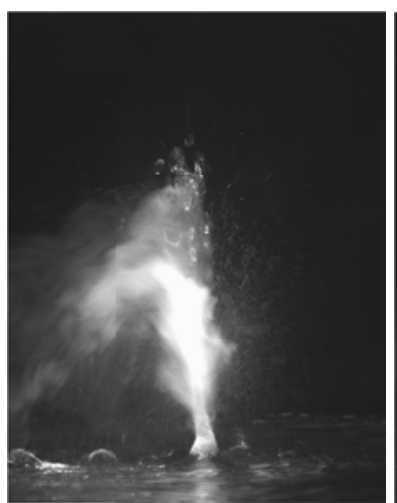

Heptane

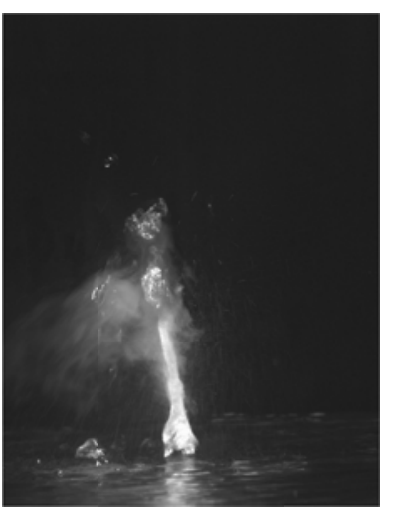

Decane

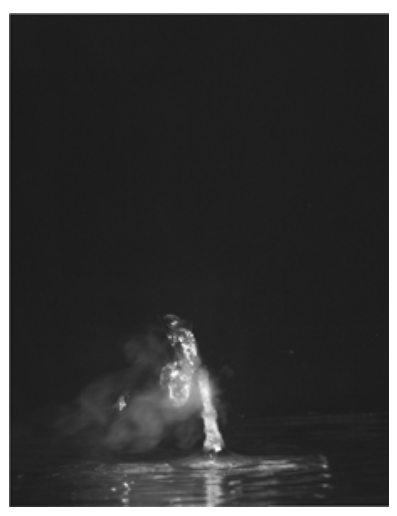

Water

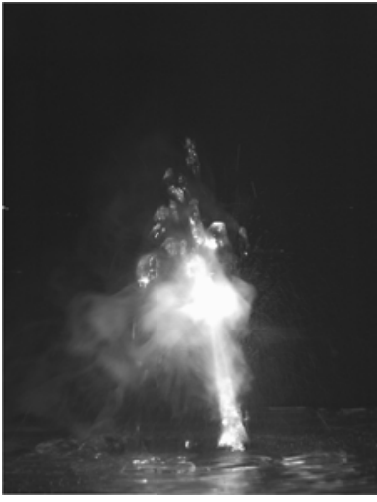

Methanol

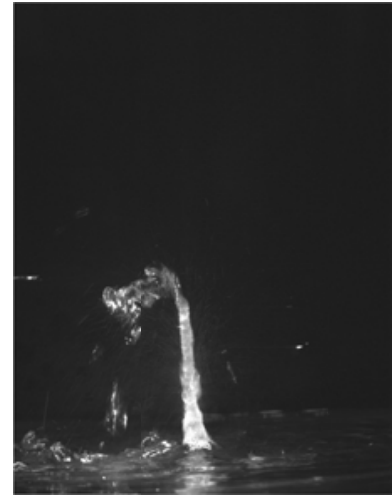

Ethanol

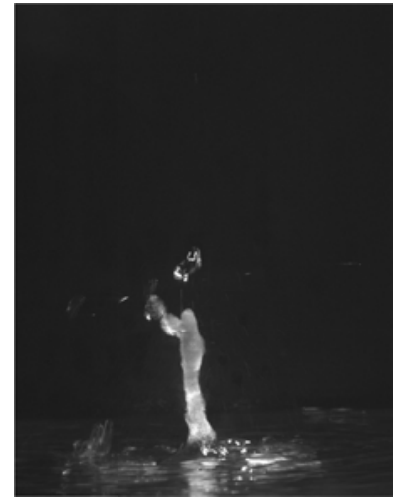

2-Propanol

Figure 6. Ultrasonic atomization of different liquids for a common forcing voltage of $30 \mathrm{~V}$ 
From the physical properties listed in Table 1, it is to be observed that the main difference between water and the rest of the liquids can be found in the values for surface tension and compressibility modulus, which are the highest. This could be a reason for its distinct behavior, but further research is required.

Arguments are still required to explain the presence of the atomization rate maximum for some of the tested liquids. Figure 7 shows some images of heptane atomization for driving voltages of $25 \mathrm{~V}, 30 \mathrm{~V}$ and $55 \mathrm{~V}$. Although for all the recorded images atomization seems to be more violent for higher forcing, what appears to increase in a higher degree is the formation of medium-sized droplets, in detriment of the production of the smallest ones. As in the present experiments the atomization rate measurements are limited to this last group of droplets, this might explain the maximum in the curves in Fig. 4.
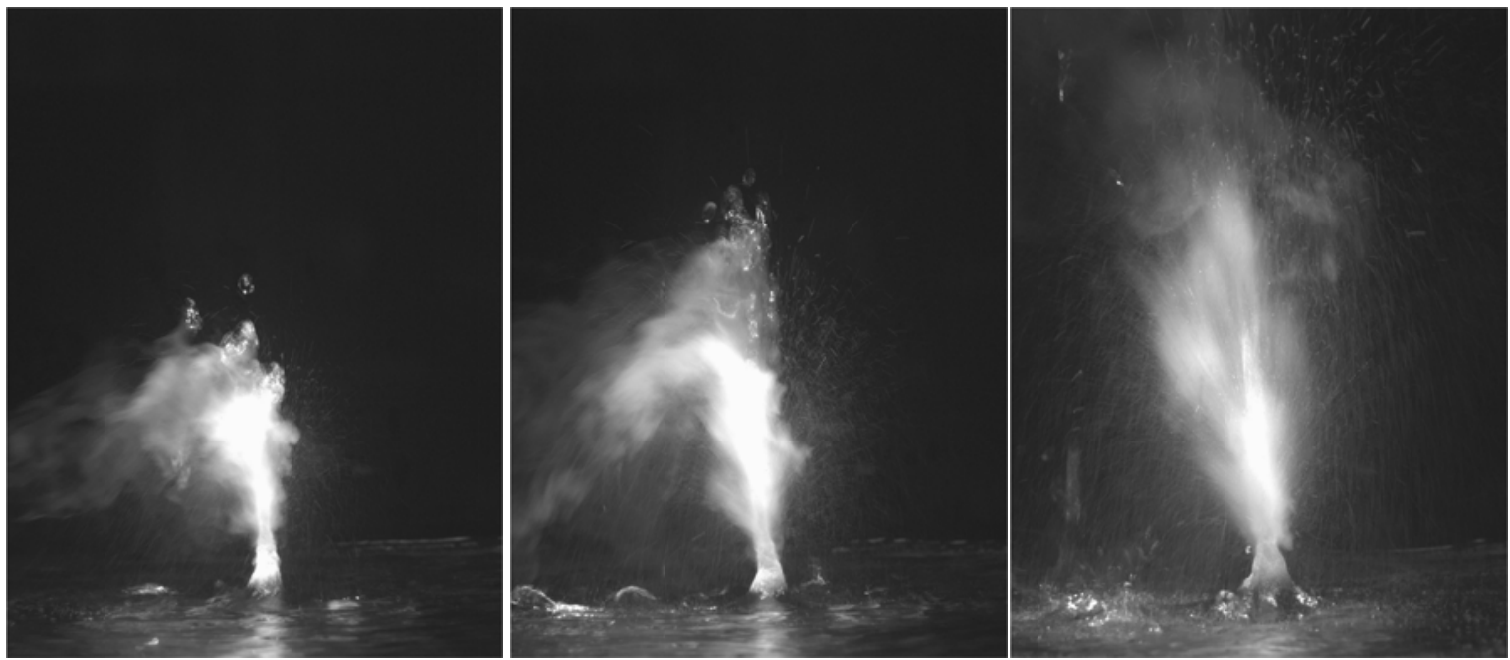

Figure 7. Ultrasonic atomization of heptane for forcing voltage values of $25 \mathrm{~V}, 30 \mathrm{~V}$ and $55 \mathrm{~V}$.

The process of droplet ejection from the cone surface is shown in Fig. 8. It corresponds to methanol atomization for a forcing voltage of $25 \mathrm{~V}$. Time interval between frames is $333 \mu \mathrm{s}$. Relatively large droplets can be seen escaping from the liquid surface. The smallest droplets cannot be discerned individually, and they appear as a fine mist. As a first estimate, this indicates that their size has to be lower than $8 \mu \mathrm{m}$. The droplet size distribution measurements should confirm this extreme.
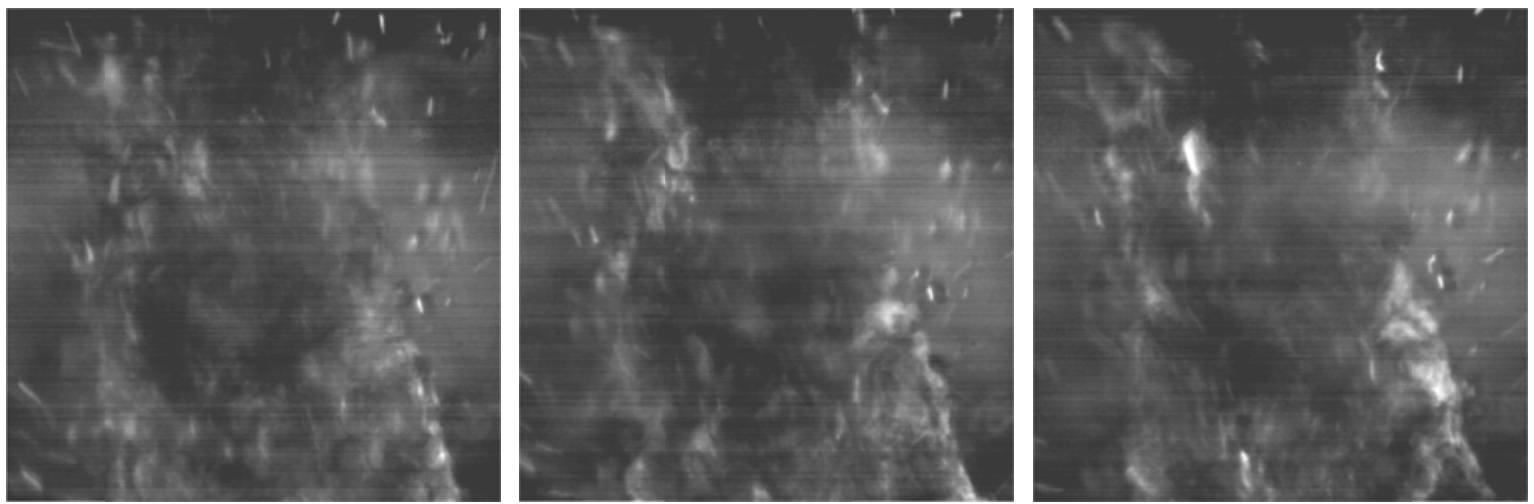

Figure 8. Close up view of ultrasonic atomization of methanol for a forcing voltage of $25 \mathrm{~V}$. Time interval between frames is 333 $\mu \mathrm{s}$.

\section{Droplet size distribution measurements}

Similarly to what happened for the atomization rate measurements, the specific experimental set up arranged to determine droplet size distribution only considers the small drops that form the mist. As they are dragged out of the recipient by a slow air flow, large droplets are effectively filtered out by effect of gravity. It is again to be reminded that large droplets are not considered of interest because they can be produced in an efficient way by a variety of atomization methods. It is the generation of micron-sized droplets what is difficult to achieve with other procedures alternative to ultrasonic atomization. 
Measurements for water were already obtained in previous works [2,8]. Some of them have been retaken to ensure repeatability. Main results are compiled in Fig. 9. As expected, it is confirmed that for the liquids in these experiments and for the droplets in the micron range, variation in their physical properties has a weak influence on the size distribution function. For most of the liquids, the shape of the distribution function is nearly identical, with two distinct peaks at $3.3 \mu \mathrm{m}$ and $6.2 \mu \mathrm{m}$. Size distributions for hexane, ethanol and propanol show a slightly different shape. It may be due to the fact that accuracy of these measurements is somewhat lower. The measurements for hexane are inaccurate because they were affected by laser beam steering due to the high concentration of vapors. Non-zero results were obtained flowing the dragging air inside the liquid container with the ultrasonic transducer disconnected. However, we were unable to separate the vapor from the droplets to be measured. Ethanol and 2-Propanol measurements are also inaccurate because droplet concentration was very low and laser obscuration was below the recommended level. Measurements for the rest of the liquids seem to confirm the hypothesis postulated in many previous papers that relates droplet size only to ultrasonic frequency.

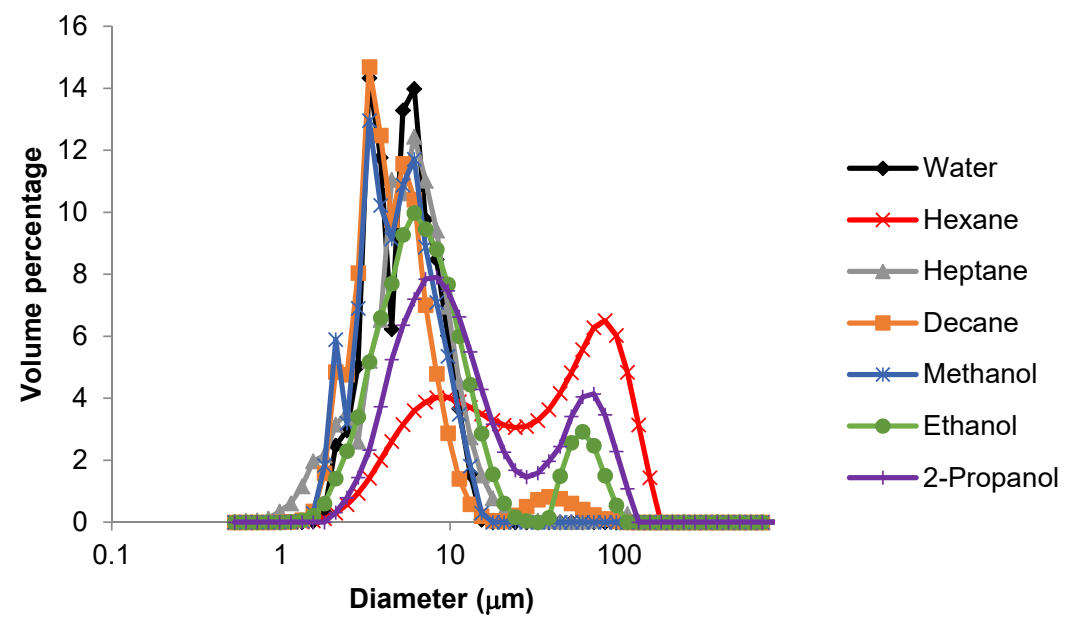

Figure 9. Droplet size distribution functions for the different atomized liquids.

\section{Conclusions}

An experimental study has been conducted to analyze the characteristics of ultrasonic atomization when working with different organic compounds. Three alkanes (hexane, heptane and decane) and three alcohols (methanol, ethanol and 2-propanol) have been considered. Density and surface tension values are quite similar for all of them, in all cases lower than those for water. Viscosity and vapor pressure cover a wider range, increasing for increasing number of $\mathrm{C}$ atoms. Tests operating with water have also been performed for comparison purposes. A commercial piezoceramic disk with a resonance frequency of $1.65 \mathrm{MHz}$, has been used in the experiments. It has been forced with a sinusoidal wave coincident with the resonance frequency and variable amplitude. The maximum applied voltage has been limited to $80 \mathrm{~V}$. In all cases, atomization rates have been measured. Sprays have been characterized analyzing instantaneous images and high speed video sequences, and measuring droplet size distribution functions. It has been observed that in the process of atomization a fine mist of small micron-sized droplets is generated, together with some medium sized droplets that are also ejected from the liquid surface. The amount of this last type of droplets and their detachment velocity clearly increase with increasing forcing voltage. In this study, attention has been only focused on the small drops that form the mist. The large droplets have not been considered of interest because they can be produced in an efficient way by a variety of atomization methods and can be easily filtered out. It is the generation of micron-sized droplets what is difficult to achieve with other procedures alternative to ultrasonic atomization. A somehow unexpected observation is that, contrary to the case of operation with water in which atomization rate always increases with voltage, alkane and alcohol atomization presents a maximum for a certain value, decreasing when voltage is further increased. The presence of this maximum can be attributed to an increase with voltage in the formation of medium-sized droplets, in detriment of the production of the smallest ones. Results also confirm that viscosity strongly conditions the atomization efficiency. On the other hand it has been verified that for a fixed ultrasonic frequency, size of the fog droplets seems to be independent of the liquid nature. 


\section{Acknowledgements}

This work has been partially funded by the Secretariat of State for Research of the Spanish Ministry of Economy and Competitiveness under project DPI2013-45814-P. Support of the Regional Government of Aragon to the Experimental Fluid Dynamics Research Group (T03) is also acknowledged.

\section{References}

[1] Wood, W.R. and Loomis, A.L., (1927), The physical and biological effects of high frequency sound-waves of great intensity, Phil. Mag., vol. 4 (22), 417-437.

[2] Barreras, F., Amaveda, H., Lozano, A., (2002), Transient High-Frequency Ultrasonic Water Atomization, Exp. Fluids, vol. 33 (3), 405-413.

[3] Avvaru, B., Patil, M.N., Gogate, P.R., Pandit, A.B., (2006), Ultrasonic atomization: Effect of liquid phase properties, Ultrasonics, 44, 146-158.

[4] Bassett, J.D. and Bright, A.W., (1976), Observations Concerning the Mechanism of Atomization in an Ultrasonic Fountain, J. Aerosol Sci., vol. 7, (1) 47-51.

[5] Donnelly T.D., Hogan J., Mugler A., Schommer, N., Schubmehl, M., Bernoff, A.J., Forrest, B., (2004), "An experimental study of micron-scale droplet aerosols produced via ultrasonic atomization", Phys. Fluids, vol. 16 (8), 2843-2851.

[6] Sindayihebura, D. and Bolle, L., (1998), Ultrasonic Atomization of Liquid: Stability Analysis of the Viscous Liquid Film Free Surface, Atomization and Sprays, vol. 8, 217-233.

[7] Rajan, R., Pandit, A.B., (2001), Correlations to predict droplets size in ultrasonic atomization, Ultrasonics, 39, 235-255

[8] Lozano, A., García, J.A., Navarro, J.L., Calvo, E., Barreras, F., (2010), Influence of viscosity on droplet size distribution and generation rate in ultrasonic atomization Atomization and Sprays, 20, (11): 923-934.

[9] Lozano, A., García, J.A., Ranz, A., Barreras, F., Santolaya, J.L., (2014), Ultrasonic atomization of mixtures and suspensions, 26th Annual Conference on Liquid Atomization and Spray Systems, Bremen, Germany, 810 Sep. 2014.

[10] Lighthill, J., Acoustic streaming, (1978), Journal of Sound and Vibration, vol. 61 (3), 391-418, 1978. 